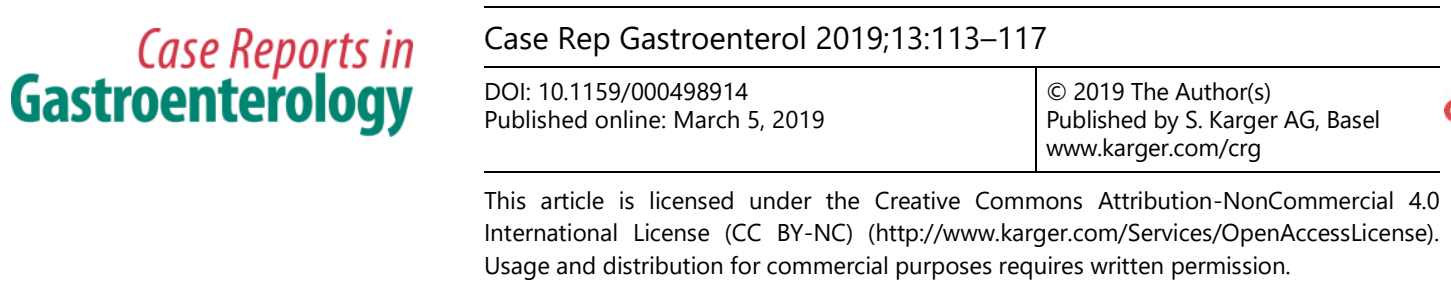

\title{
Successful Endoscopic Removal of a Biliary Stent with Stent-Stone Complex after Long-Term Migration
}

\author{
Masashi Morimachi Masami Ogawa Masashi Yokota Aya Kawanishi \\ Yohei Kawashima Tetsuya Mine \\ Department of Gastroenterology and Hepatology, Tokai University School of Medicine, \\ Kanagawa, Japan
}

\section{Keywords}

Migrated biliary stent $\cdot$ Stent-stone complex . Endoscopic retrograde cholangiopancreatography

\begin{abstract}
A 49-year-old man was referred to our hospital for an abnormality of the hepatobiliary enzyme. The patient was diagnosed with primary sclerosing cholangitis 9 years ago, and he had a biliary stent with a string placed as an inside stent. We attempted to remove the stent 6 months later but the string was cut off, so the stent could not be removed. Removal was attempted again, but the patient cancelled the outpatient appointments. During the examination performed at the present visit, we discovered that the biliary stent had migrated into the bile duct, and a stone had formed around the stent. We attempted to remove the stent-stone complex by endoscopic retrograde cholangiopancreatography, but it was difficult; thus, we decided to implant a new biliary stent and remove the other stent later. When we performed endoscopic retrograde cholangiopancreatography again 2 days later, the bile duct axis was linearized thanks to the additional stent, enabling us to grab the migrated stent with stent-stone complex using grasping forceps and to successfully pull it out. By implanting an additional plastic stent temporarily, we were able to straighten the biliary axis and endoscopically remove the biliary stent that migrated and caused the development of stent-stone complex in a 2-staged approach.




\section{Case Reports in Gastroenterology}

Case Rep Gastroenterol 2019;13:113-117 DOI: $10.1159 / 000498914$

(c) 2019 The Author(s). Published by S. Karger AG, Basel www.karger.com/crg

Morimachi et al.: Successful Endoscopic Removal of a Biliary Stent with Stent-Stone Complex after Long-Term Migration

\section{Introduction}

Endoscopic biliary stenting for biliary stricture is an effective and widely accepted treatment. However, it may be accompanied by procedural complications, such as bleeding and perforation, and problems with the stent, such as deviation, migration, and occlusion. There are plastic and metallic biliary stents. The metallic stents are used for inoperable or postoperative recurrence of malignant tumors. The plastic stents (PSs) are generally used for benign diseases and operable malignant tumors. The rate of PS migration into the bile duct is 1.7$10 \%$ [1-3], and the success rate of endoscopic removal of a migrated stent is $80-90 \%$ [3-5]. If the stent cannot be endoscopically removed, percutaneous treatment may be performed, or surgical removal might become necessary [5, 6]. Presently, there is no special procedure or device for endoscopic removal of a PS that migrates into the bile duct or pancreatic duct; thus, each operator has to attempt endoscopic removal with existing tools [4]. In 2007, Tang et al. [7] reported stent-stone complex (SSC) as a complication of biliary stent implantation. SSC is a rare procedural complication associated with the long-term implantation of a biliary stent, which leads to the formation of a stone around the stent. SSC can make endoscopic removal of the stent difficult. There are few reports of biliary stent migration into the bile duct with SSC. Herein, we describe a case of successful endoscopic removal of a biliary stent with SSC after long-term migration.

\section{Case Report}

A 49-year-old man was referred to our hospital for an abnormality of the hepatobiliary enzyme. The patient had no subjective symptom. He was diagnosed with right intrahepatic biliary dilation 9 years ago in another hospital and was examined in our department during the same year. At that time, the patient was also diagnosed with primary sclerosing cholangitis. Underlying conditions included hyperlipidemia and chronic renal insufficiency due to nephrosclerosis. The only oral medication administered was pravastatin sodium. Without endoscopic sphincterotomy, a biliary tube stent with a string had been placed as an inside stent to treat biliary stricture. Six months later, we attempted to remove the stent, but since the string was cut off, the attempt was abandoned. Removal was rescheduled for a later date, but the patient cancelled subsequent outpatient appointments.

At the present evaluation, vital signs were stable, and there were no notable physical findings. He did not have abdominal pain or jaundice. The blood test results confirmed an elevated inflammatory reaction (white blood cell count 8,700/ $\mu \mathrm{L}$ and C-reactive protein level 10.0 $\mathrm{mg} / \mathrm{dL}$ ) and liver function (alanine transaminase level $37 \mathrm{U} / \mathrm{L}$, aspartate transaminase level $48 \mathrm{U} / \mathrm{L}$, alkaline phosphatase level 2,661 U/L, $\gamma$-glutamyl transpeptidase level $742 \mathrm{U} / \mathrm{L}$, total bilirubin level $3.7 \mathrm{mg} / \mathrm{dL}$, and direct bilirubin level $2.7 \mathrm{mg} / \mathrm{dL}$ ). Abdominal X-ray showed that the stent remained in the bile duct and a stick-like stone had formed around the stent. Abdominal ultrasonography and abdominal plain computed tomography showed extension of the intrahepatic bile duct and SSC. Therefore, the patient was diagnosed with cholangitis due to occlusion of the residual stent, and he was hospitalized for stent and SSC removal with endoscopic retrograde cholangiopancreatography (ERCP).

On the first day of hospitalization, we performed ERCP. For sedation, we used pethidine hydrochloride (35 mg) and midazolam ( $2 \mathrm{mg}$ ). For the endoscope, we used the JF $260 \mathrm{~V}$ (Olympus). As the endoscope was inserted, it became clear that there was no string at the major duodenal papilla, and the SSC was completely embedded into the bile duct (Fig. 1a, b). 


\section{Case Reports in Gastroenterology}

Case Rep Gastroenterol 2019;13:113-117 DOI: $10.1159 / 000498914$

(c) 2019 The Author(s). Published by S. Karger AG, Basel www.karger.com/crg

Morimachi et al.: Successful Endoscopic Removal of a Biliary Stent with Stent-Stone Complex after Long-Term Migration

Cannulation was performed, and a contrast agent was injected. We succeeded in obtaining contrast imaging at a distal position from the SSC and in inserting a guide wire. Next, we performed endoscopic sphincterotomy and succeeded in grasping the SSC with an 8-wire basket under the guide wire, but removal was unsuccessful regardless of how we operated the endoscope. Subsequently, we attempted removal with grasping forceps, snares, and a balloon catheter, but all attempts were unsuccessful. As the procedure duration became long and it became difficult to keep the patient still, we placed a new PS (7 French, $7 \mathrm{~cm}$ ) for drainage and discontinued the procedure.

After the procedure, there was no change in the patient's subjective symptoms or blood test results. Two days later, we attempted ERCP again under the same conditions. The new PS that had been inserted previously straightened the biliary axis (Fig. 2a, b); thus, we were able to directly insert the grasping forceps into the bile duct, grasp the lower edge of the SSC, and easily remove it with the stent by pulling it out via the endoscope (Fig. 3a, b). Then, we removed the PS that was placed during the prior ERCP, confirmed that there was no residual stone using the offset balloon catheter, and completed the procedure. After the procedure, we confirmed that there was no complication, and the enzyme of the biliary system was improved.

The patient was discharged after 5 days of hospitalization. Twenty-four months since discharge, there has been no recurrence of cholangitis.

\section{Discussion}

Complications associated with endoscopic biliary stenting include stent migration, which occurs occasionally. To remove the migrated stent, a basket catheter, snare, balloon catheter, grasping forceps, stent retriever, and other tools can be used. During the first ERCP, we were unable to remove the migrated stent. The causes for unsuccessful removal of a migrated stent include the upper end of the PS being embedded into the bile duct bifurcation, the lower end of the PS being embedded into the bile duct wall, or stricture near the lower end of the PS. In the present case, the lower end of the PS was embedded into the bile duct wall, and a stone formed around the stent, making endoscopic removal difficult. We placed a new PS during the first ERCP, which led to the straightening of the biliary axis, causing the lower end of the SSC to become separated from the bile duct wall, allowing for its removal during the second ERCP. Fortunately, the fact that the SSC was a stick-like type stone, not a dumbbell type stone, was another reason why we were able to remove it without resistance. A long procedure time increases the probability of complications; thus, when stent recovery is difficult, it is effective to place a drainage tube in the bile duct and retry removal at a later date. In our facility, we use 60 min or when sedation of the patient becomes difficult as the guidelines for discontinuing the procedure.

Herein, we encountered a rare condition in which a biliary stent migrated into the bile duct, causing the development of SSC. When we searched "stent stone complex" and "stone around stent" in PubMed, there were 14 cases in which detailed removal methods were described. In the literature, the treatment methods varied and ranged from simple grasping forceps and a balloon catheter to the use of a cholangioscope, electro-hydraulic lithotripsy or extracorporeal shockwave lithotripsy, and multi-phase procedures. Reports even included cases in which the stent could not be removed, and the procedure was finished by implanting an additional stent [8] or performing surgical removal [9]. 
Although endoscopic biliary stenting is relatively simple and widely used as an effective treatment, it can lead to unexpected complications. There is no designated device for treating the many complications of biliary stenting; therefore, each operator has to approach the situation through trial and error. In the present study, by implanting an additional PS temporarily, we were able to straighten the biliary axis and endoscopically remove the biliary stent that migrated and caused the development of an SSC in a 2-staged approach.

\section{Statement of Ethics}

Written informed consent for publication was obtained from the patient.

\section{Disclosure Statement}

The authors have no conflicts of interest to declare.

\section{Funding Sources}

There were no funding sources.

\section{Author Contributions}

Masashi Morimachi wrote this paper, and all other authors equally contributed to the patient's medical treatment.

\section{References}

1 Johanson JF, Schmalz MJ, Geenen JE. Incidence and risk factors for biliary and pancreatic stent migration. Gastrointest Endosc. 1992 May-Jun;38(3):341-6.

2 Mueller PR, Ferrucci JT Jr, Teplick SK, vanSonnenberg E, Haskin PH, Butch RJ, et al. Biliary stent endoprosthesis: analysis of complications in 113 patients. Radiology. 1985 Sep;156(3):637-9.

3 Tarnasky PR, Cotton PB, Baillie J, Branch MS, Affronti J, Jowell P, et al. Proximal migration of biliary stents: attempted endoscopic retrieval in forty-one patients. Gastrointest Endosc. 1995 Dec;42(6):513-20.

4 Chaurasia OP, Rauws EA, Fockens P, Huibregtse K. Endoscopic techniques for retrieval of proximally migrated biliary stents: the Amsterdam experience. Gastrointest Endosc. 1999 Dec;50(6):780-5.

5 Lahoti S, Catalano MF, Geenen JE, Schmalz MJ. Endoscopic retrieval of proximally migrated biliary and pancreatic stents: experience of a large referral center. Gastrointest Endosc. 1998 Jun;47(6):486-91.

6 Price LH, Brandabur JJ, Kozarek RA, Gluck M, Traverso WL, Irani S. Good stents gone bad: endoscopic treatment of proximally migrated pancreatic duct stents. Gastrointest Endosc. 2009 Jul;70(1):174-9.

7 Tang SJ, Armstrong L, Lara LF, Kortan P. De novo stent-stone complex after long-term biliary stent placement: pathogenesis, diagnosis, and endotherapy. Gastrointest Endosc. 2007 Jul;66(1):193-200.

8 Schuller AM, Rezk GJ, Lyon DT. Calculus formation around common bile duct stents: a complication of longterm biliary drainage. Gastrointest Endosc. 1991 Sep-Oct;37(5):581-2.

9 Barai V, Hedawoo J, Changole S. Forgotten CBD stent (102 months) with stone-stent complex: A case report. Int J Surg Case Rep. 2017;30:162-4. 


\section{Case Reports in Gastroenterology}
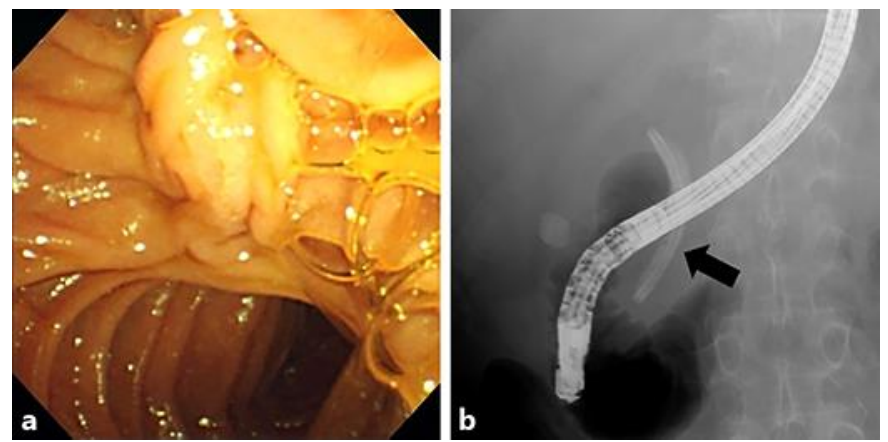

Fig. 1. Endoscopic retrograde cholangiopancreatogram on the first day of hospitalization. There is no string or stent at the major duodenal papilla (a). The X-ray shows the stent remaining in the bile duct, and a sticklike stone has formed around the stent (b, arrow).
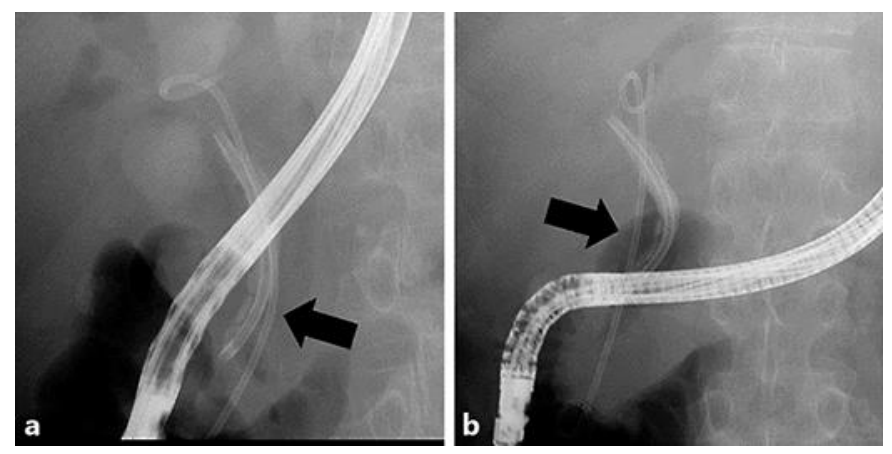

Fig. 2. These images show a comparison between placement of the new plastic stent (PS) (a, arrow) and the same PS 2 days later (b, arrow). The newly added PS has straightened the bile duct, and the lower end of the stent-stone complex is separated from the bile duct wall.

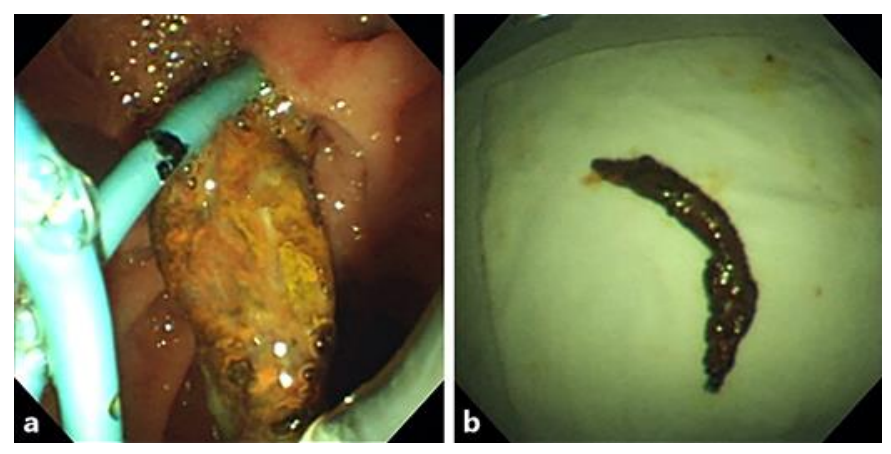

Fig. 3. These images show the stent-stone complex (SSC) being grasped with grasping forceps and pulled out (a). The length of the removed SSC is about $4 \mathrm{~cm}$ (b). 